\title{
The grand challenge of quantum computing: bridging the capacity gap
}

\author{
Alexandre Zagoskin* \\ Loughborough University, Loughborough, UK \\ ${ }^{*}$ Correspondence: a.zagoskin@lboro.ac.uk \\ Edited and reviewed by: \\ Tobias Lindstrom, National Physical Laboratory, UK
}

Keywords: Quantum computing, quantum simulation, quantum engineering, testing limits of applicability of quantum mechanics, quantum metamaterials

The fabrication and control of macroscopic artificial quantum structures, such as qubits (Mooij et al., 1999; Nakamura et al., 1999; Friedman et al., 2000), qubit arrays (Johnson et al., 2011; Barends et al., 2014), quantum annealers (Boixo et al., 2013) and, recently, quantum metamaterials (Macha et al., 2014), have witnessed significant progress over the last 15 years. This was a surprisingly quick evolution from theoretical musings to what can now be called quantum engineering [the observation of such phenomena even in a single superconducting device was considered a truly challenging task in 1980 (Leggett, 1980)]. And today, we stand at the point where existing theoretical and computational tools become inadequate for predicting, analyzing, and simulating the behavior of such structures, in which quantum superposition and entanglement play the key role (Zagoskin et al., 2014).

The long-known fundamental impossibility of simulating large enough quantum systems by classical means (Feynman, 1982), unfortunately, manifests itself already at the level of systems containing as few as several hundreds of qubits. Such a system is still too small to be used as an efficient quantum simulator of comparable systems, but already too large for us to tell with certainty, using the existing classical tools, whether it behaves as a quantum system should (Smolin and Smith, 2014). Furthermore, the complexity of already existing quantum processor prototypes confronts us with an engineering problem designing a reliable quantum device and testing its reliability.

What is even worse, if there are fundamental corrections to the laws of quantum mechanics for large enough systems, we will be unable to discover them because of our inability to tell what exactly quantum mechanics would predict.

Let us take the optimistic view that quantum computing is not fundamentally restricted by, for example, the size of a system capable of demonstrating quantum behavior (Penrose, 1999). In this scenario, it would be possible to create quantum computing devices that will allow us to design and fabricate ever bigger and better quantum computers, as well as other macroscopic quantum devices, of a character and use of which we cannot even imagine at the moment. Alternatively, we may find fundamental limits to the applicability of quantum mechanics. Nevertheless, this can happen only if the gap between our current ability to characterize large quantum systems and the capacities of the smallest workable quantum computers is bridged.

Bridging this capacity gap is thus the immediate grand challenge for the field: a challenge that must be met if we hope to make further progress in quantum computing and quantum engineering or if we hope to discover fundamentally new physics, or both.

While it is impossible to efficiently simulate a large quantum system by classical means by directly solving the appropriate equations of motion, it is feasible that essential quantum properties of an ensemble of such systems will be reflected in certain higher-level, global characteristics. These properties should be insensitive to details of a particular instance, computable by classical tools and accessible to experimental investigation.

This view of a system of qubits as a quantum many-body system should be amenable to the approaches that have proven to work very well in numerous applications in condensed matter physics and quantum statistical mechanics.

Therefore, with such earlier breakthroughs in mind, the task at hand will be difficult yet not impossible, and more than worth the effort.

\section{ACKNOWLEDGMENTS}

I am grateful to J. Betouras, M. Everitt, A. Miranowicz, and J. H. Samson for stimulating discussions and helpful criticisms.

\section{REFERENCES}

Barends, R., Kelly, J., Megrant, A., Veitia, A., Sank, D., Jeffrey, E., et al. (2014). Superconducting quantum circuits at the surface code threshold for fault tolerance. Nature 508, 500-503. doi:10.1038/ nature 13171

Boixo, S., Albash, T., Spedalieri, F., Chancellor, N., and Lidar, D. (2013). Experimental signature of programmable quantum annealing. Nat. Commun. 4, 2067. doi:10.1038/ncomms3067

Feynman, R. (1982). Simulating physics with computers. Int. J. Theor. Phys. 21, 467-488. doi:10.1007/ BF02650179

Friedman, J., Patel, V., Chen, W., Tolpygo, S., and Lukens, J. (2000). Quantum superposition of distinct macroscopic states. Nature 406, 43-46. doi: 10 . 1038/35017505

Johnson, M. W., Amin, M. H., Gildert, S., Lanting, T., Hamze, F., Dickson, N., et al. (2011). Quantum annealing with manufactured spins. Nature 473, 194-198. doi:10.1038/nature10012

Leggett, A. (1980). Macroscopic quantum systems and the quantum theory of measurement. Suppl. Prog. Theor. Phys. 69, 80.

Macha, P., Oelsner, G., Reiner, J.-M., Marthaler, M., André, S., Schön, G., et al. (2014). Implementation of a quantum metamaterial using superconducting qubits. Nat. Commun. 5, 5146. doi:10.1038/ ncomms6146

Mooij, J. E., Orlando, T. P., Levitov, L., Tian, L., van der Wal, C. H., and Lloyd, S. (1999). Josephson persistent-current qubit. Science 285, 1036-1039. doi:10.1126/science.285.5430.1036

Nakamura, Y., Pashkin, Y., and Tsai, J. (1999). Coherent control of macroscopic quantum states in a 
single-Cooper-pair box. Nature 398, 786-788. doi: $10.1038 / 19718$

Penrose, R. (1999). The Emperor's New Mind. Oxford: Oxford University Press.

Smolin, J. A., and Smith, G. (2014). Classical signature of quantum annealing. Front. Phys. 2:52. doi:10.3389/fphy.2014.00052

Zagoskin, A. M., Il'ichev, E., Grajcar, M., Betouras, J. J., and Nori, F. (2014). How to test the "quantumness" of a quantum computer? Front. Phys. 2:33. doi:10.3389/fphy.2014.00033
Conflict of Interest Statement: The author declares that the research was conducted in the absence of any commercial or financial relationships that could be construed as a potential conflict of interest.

Received: 24 September 2014; accepted: 09 October 2014; published online: 22 October 2014.

Citation: Zagoskin A (2014) The grand challenge of quantum computing: bridging the capacity gap. Front. ICT 1:2. doi: 10.3389/fict.2014.00002
This article was submitted to Quantum Computing, a section of the journal Frontiers in ICT.

Copyright (c) 2014 Zagoskin. This is an open-access article distributed under the terms of the Creative Commons Attribution License (CC BY). The use, distribution or reproduction in other forums is permitted, provided the original author(s) or licensor are credited and that the original publication in this journal is cited, in accordance with accepted academic practice. No use, distribution or reproduction is permitted which does not comply with these terms. 